Int. J. Dev. Biol. 55: 975-979

doi: $10.1387 /$ ijdb.103279fs

\title{
Effect of the transformer-2 gene of Anastrepha on the somatic sexual development of Drosophila
}

\author{
FRANCESCA SARNO, MARÍA-FERNANDA RUIZ\# and LUCAS SÁNCHEZ*,\# \\ Centro de Investigaciones Biológicas (C.S.I.C.), Madrid, Spain
}

\begin{abstract}
The transformer-2 gene is involved in sex determination in tephritid flies (Tephritidae). It is required for the auto-regulation of the transformer gene (the memory device for sex determination in these insects) and for the female-specific splicing of doublesex pre-mRNA, the last gene in the sex determination gene cascade. The present manuscript addressed the question of the functional conservation of the tephritid AnastrephaTra2 protein to direct sexual development in Drosophila (Drosophilidae). To express this protein in Drosophila, the GAL4-UAS system was used. The Anastrepha Tra2 protein supplies tra-2 function in Drosophila: this protein would form a complex with the endogenous Drosophila Tra protein to promote the female-specific splicing of the Drosophila doublesex pre-mRNA. The feminisation produced by the Anastrepha Tra2 protein was, however, partial.
\end{abstract}

KEY WORDS: Anastrepha, Drosophila, sex determination, transformer-2

The characterisation of sex determination genes in Drosophila Melanogaster has shown that the product of a gene controls the sex-specific splicing of the pre-mRNA from the downstream gene in the genetic cascade (reviewed in Sánchez et al., 2005). Sex-lethal $(S X I)$ is at the top of this cascade and acts as the memory device for female sexual development via its auto-regulatory function: its product controls the splicing of its own pre-mRNA (Bell et al., 1991). In addition, Sxl controls the splicing of the pre-mRNA from the downstream gene transformer (tra) (Boggs et al., 1987; Belote et al., 1989). The Tra product and the product of the constitutive gene transformer-2 (tra-2) (Goralski et al., 1989; Amrein et al., 1990) control the sex-specific splicing of the pre-mRNA of the gene doublesex (dsx), which is transcribed in both sexes (Burtis and Baker 1989). In females, the Tra-Tra2 complex binds to the female-specific exon (see Fig. 2A) and directs the splicing of the $d s x$ pre-mRNA according to the female mode, giving rise to the female DsxF protein that promotes female sexual development. In males, in which no functional Tra protein is available, the $d s x$ pre-mRNA follows the default male mode of splicing, giving rise to the mature $d s x M$ mRNA, which produces male DsxM protein (Burtis and Baker 1989; Hoshijima et al., 1991; Hedley and Maniatis 1991; Ryner and Baker 1991; Tian and Maniatis 1993; Hertel et al., 1996). This promotes male sexual development.

Genes homologous to the sex determination genes of $D$. melanogaster have been sought in other insects (reviewed in Sánchez 2008; Verhulst et al., 2010; Gempe and Beye 2010). In the tephritid fruit flies, the $S x /$ gene is not regulated in a sex-specific fashion (Saccone et al., 1998; Lagos et al., 2005) so that in the tephritids, Sxl does not appear to play the key discriminating role in sex determination that it plays in Drosophila. As in the drosophilids, the tephritid tra (Pane et al., 2002; Lagos et al., 2007; Ruiz et al., 2007) and tra-2 (Salvemini et al., 2009; Sarno et al., 2010) genes are expressed in both sexes. The tra primary transcript shows sexspecific alternative splicing. However, whereas in the drosophilids $S x /$ regulates tra, in the tephritids this gene appears to have an auto-regulatory function that produces functional Tra protein specifically in females (Pane et al., 2002; Lagos et al., 2007; Ruiz et al., 2007) so that it plays the key regulatory memory device for sex determination (Pane et al., 2002). A similar role for tra has been observed in the dipterans Musca domestica (Muscidae) (Hediger et al., 2010) and in Lucilia cuprina (Calliphoridae) (Concha and Scott 2009). The injection of the respective tra-2 dsRNA into Musca (Burghardt et al., 2005), Ceratitis (Salvemini et al., 2009) and Anastrepha (Sarno et al., 2010) results in the destruction of endogenous tra-2 function in these species and the subsequent male-specific splicing of the endogenous tra and $d s x$ pre-mRNAs, leading to the transformation of genotypically female embryos into adult pseudomales. This highlights the role of tra-2 in Musca, Ceratitis and Anastrepha sex determination.

Abbreviations used in this paper: Tra-2, transformer-2.

\footnotetext{
*Address correspondence to: Lucas Sánchez. Centro de Investigaciones Biológicas, Ramiro de Maeztu 9, E-28040 Madrid, Spain. Tel: +34-91-837-3112. Fax: +34-91-536-0432. E-mail: Isanchez@ cib.csic.es - \#Note: The indicated authors are co-senior authors.
} 
The present paper studied whether the Anastrepha Tra2 protein shows conserved sex-determination function in Drosophila; i.e., whether Anastrepha Tra2 is able to substitute the endogenous Drosophila Tra2 protein in the control of sex determination in this species.

\section{Results and Discussion}

The rationale of the experiment was to express the transgenic AoTra2 protein in Drosophila XX pseudomales lacking the tra-2 gene function and checked whether these pseudomales showed feminisation. The GAL4-UAS system was used to express the AoTra2 protein in Drosophila.

The systemic expression of AoTra2 with the ubiquitous-expression da-GAL4 or hs-GAL4 drivers was found to be lethal to both male and female flies. The same lethality has been observed in Drosophila males and females that ectopically express their own Tra2 protein (Qi et al., 2006). Therefore, the rn-GAL4 local expression driver was used. This driver expresses GAL4 in agreement with the expression domain of the gene rotund $(r n)$, which is expressed in imaginal discs as well as in the embryonic and larval central nervous systems (CNS) (St. Pierre et al., 2002). The expression of $r n$ in the tarsal region of the foreleg imaginal disc commences during the early third larval instar, but is no longer evident in the late third instar, though in the rn-GAL4 line persists the tarsal expression (St. Pierre et al., 2002). This $r n-G A L 4$ driver allowed the expression of the AoTra2 protein in the foreleg basitarsus, a well-characterised sexually dimorphic region of Drosophila. The expression of AoTra2 by the rn-GAL4 driver was also lethal to both males and females. This might be due to the expression of AoTra2 in the embryonic and/or larval CNS, as mentioned above.

To prevent embryonic lethality a strategy was followed that allowed the temporal control of AoTra2 expression under the rnGAL4 driver. For this purpose the GAL4/GAL80 system was used. The GAL80 protein inhibits GAL4 function. GAL80 is temperature sensitive, with $18^{\circ} \mathrm{C}$ the most permissive temperature and $29^{\circ} \mathrm{C}$ the most restrictive (McGuire et al., 2003). Drosophila XX pseudomales mutant for tra-2 and carrying Aotra-2-UAS together with $r n-G A L 4$ and Tub-GAL80ts were produced. The cross producing these pseudomales was performed at $18^{\circ} \mathrm{C}$, and several two-days egg collections were made. Three days later each collection was transferred to $29^{\circ} \mathrm{C}$ environment. By this time the larvae had hatched, which were maintained at this temperature for the rest of their development. This treatment eliminated the embryonic lethality caused by the expression of the AoTra2 protein. Even under these conditions, the males and females expressing the AoTra2 protein were lethally affected, probably because of an excess of this transgenic protein affecting the development of the larval CNS.

Finally, since at $25^{\circ} \mathrm{C} \mathrm{GAL} 80^{\text {ts }}$ retains some function (McGuire et al., 2003), it was reasoned that if XX pseudomales mutant for tra-2 and carrying Aotra-2-UAS, rn-GAL4 and Tub-GAL80ts can develop at that temperature, the GAL4 from the $r n-G A L 4$ driver ought not to be completely inhibited. Consequently a certain amount of AoTra2 protein ought to be produced (less than at $29^{\circ} \mathrm{C}$ ) but which might not be lethal. Thus, these pseudomales might be able to survive to adulthood. This was the case. Fig. 1 shows the effect of expressing the AoTra2 protein on the sexually dimorphic development of the foreleg basitarsus in XX pseudomales and in their brother $\mathrm{XY}$ males, both mutant for tra-2 and carrying Aotra2-UAS, rn-GAL4
A
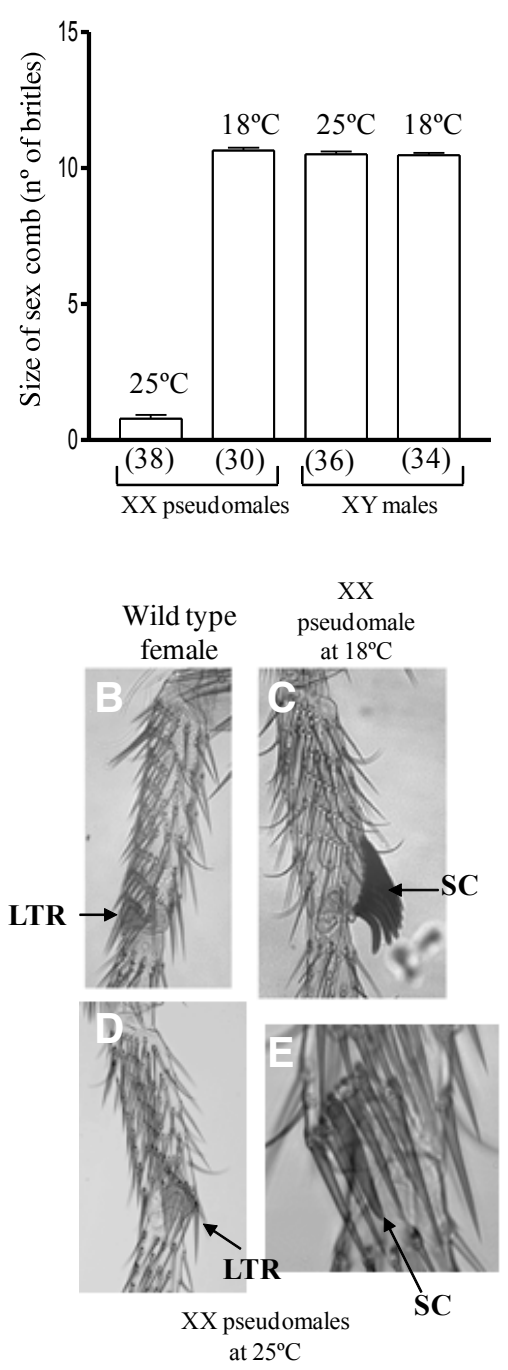

Fig. 1. Effect of the Aotra2 transgene on the somatic development of Drosophila. (A) Size (number of bristles) of the sex comb in the Aotra2 transgenic Drosophila flies. The bars in the histograms represent the 95\% confidence limits. The sample size is given in parenthesis underneath the histogram. XX pseudomales: ywTDAo\#1/w; tra-2B/Df(2R)trix, tra-2[-]; Tub-GAL80ts,w+/ rn-GAL4,w[+]. XY males: ywTDAo\#1/Y; tra-2B/Df(2R) trix,tra-2[-]; Tub-GAL80ts,w[+]/ rn-GAL4,w[+](Cross I in Materials and Methods). (B) Foreleg basitarsus of wild type female. LTR stands for last transversal row. (C) Foreleg basitarsus of XX pseudomale mutant for tra-2 at $18^{\circ} \mathrm{C}$. SC stands for sex comb. (D,E) Foreleg basitarsus of XX pseudomales mutant for tra- 2 at $25^{\circ} \mathrm{C}$.

and Tub-GAL80ts. The foreleg basitarsus contained several transversal rows, the last one forming the sex comb structure (SC) in males and in XX pseudomales mutants for tra-2 (Fig. 1C). This is composed of dark, thick bristles, and is rotated to lie parallel to the proximal-distal leg axis. Females lack the sex comb (Fig. 1B). A significant reduction ( $P<0.0001$, one-way ANOVA) was seen in the number of bristles forming the male sex comb structure in the foreleg basitarsus of $\mathrm{XX}$ pseudomales raised at $25^{\circ} \mathrm{C}$ (expressing the AoTra2 protein) compared to those raised at $18^{\circ} \mathrm{C}$ (no expression of the AoTra2 protein) (Fig. 1A). Examples are shown in Fig. $1 \mathrm{D}, \mathrm{E}$. In some cases, no sex comb-like bristles were found, yet the last transverse row (LTR) was partially or completely rotated 
resembling the location of the sex comb (Fig. 1D). In other cases, a sex comb-like bristle was found between the female-like bristles that form the last transversal row (arrow in Fig. 1E). The sex comb size of pseudomales raised at $18^{\circ} \mathrm{C}$ was the same as the sex comb of their $X Y$ brothers whether raised at 18 or $25^{\circ} \mathrm{C}(\mathrm{Fig} .1 \mathrm{~A})$.

The presence of endogenous Drosophila DsxF protein is expected to cause this reversion of the male towards the female phenotype. The analysis of the Drosophila dsxpre-mRNAsplicing in transgenic Drosophila XX pseudomales mutant for tra-2 and expressing the AoTra2 protein confirmed this expectation. The inducible hs-GAL4 driver was used to express the Aotra2transgene. XX pseudomales yw/w; Df(2R)trix, tra2[-]/tra-2B; Aotra2/hs-GAL4 were produced at $25^{\circ} \mathrm{C}$. After the hatching of the adults the flies were divided into two populations; one was maintained at $25^{\circ} \mathrm{C}$ (control flies) and one subjected to heat-shock pulses (experimental flies) to induce the expression of the Aotra2 transgene. At $25^{\circ} \mathrm{C}$, the transgenic lines did not express the female $d s x$ mRNA isoform (Fig. 2B). After the heat shocks, however, these transgenic lines expressed the female $d s x$ mRNA isoform (Fig. 2B). Two amplicons could be detected. The smaller one (646 bp) corresponded to the female $d s x F$ mRNA. The larger amplicon (758 bp) was to be expected if the intron 3 were retained (Fig. $2 \mathrm{~B})$. The cloning and sequencing of both fragments confirmed these suppositions. These results

A

$$
\text { D. melanogaster dsx gene }
$$

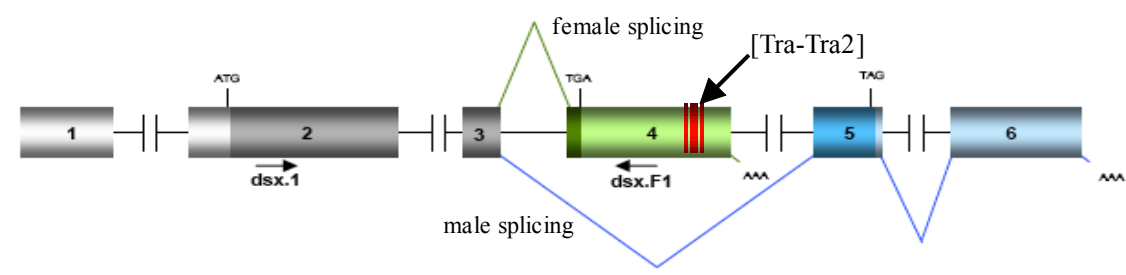

B
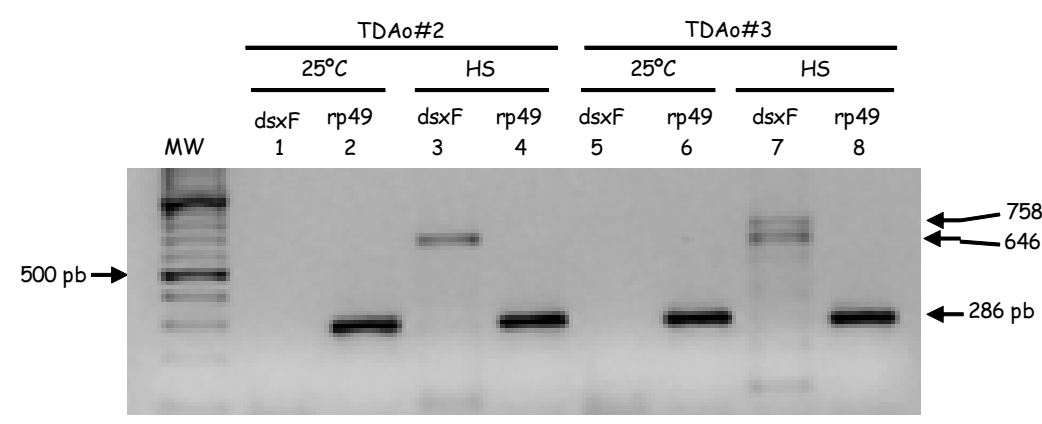

C

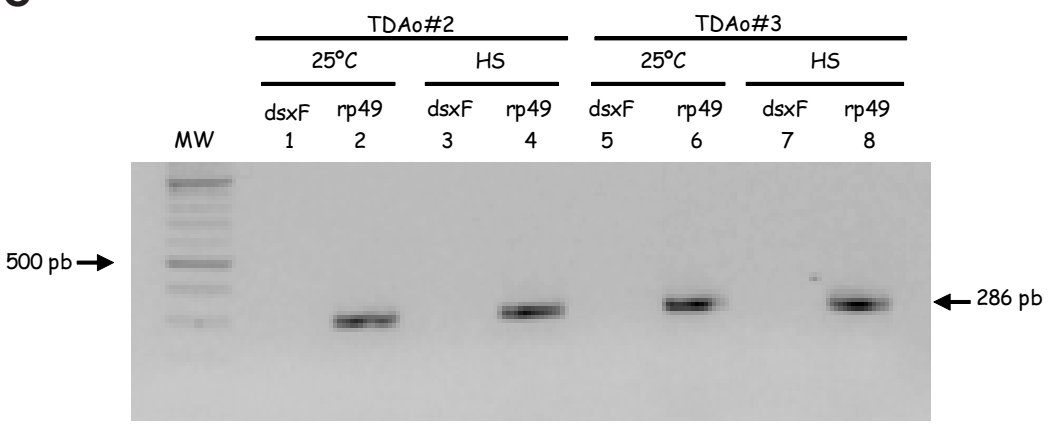

were not the consequence of the heat-shocks since their brothers (males yw/Y; Df(2R)trix,tra2[-]/tra-2B;Aotra2/hs-GAL4) expressing the Aotra2transgene did not express the female $d s x$ mRNA isoform (Fig. 2C). Negative controls for all these PCR reactions produced no amplicons (see Materials and methods). Thus, the Anastrepha Tra2 protein is able to promote the female-specific splicing of the Drosophila dsx pre-mRNA.

Whereas the expression of Anastrepha Tra2 protein in the XX pseudomales produced their feminisation, its expression in their $X Y$ normal brothers mutant for tra-2 did not affect their normal male development. This different effect is explained by the presence of Tra protein in the $X X$ pseudomales and its absence in $X Y$ normal males. It should be remembered that the female-specific dsx pre-mRNA splicing mode requires the binding of the Tra-Tra2 complex to the female-specific exon 4 (see Fig. 2A). Therefore, the Anastrepha Tra2 protein supplies tra-2 function in Drosophila: this protein would form a complex with the endogenous Drosophila Tra protein to promote the female-specific splicing of the Drosophila dsx pre-mRNA.

The feminisation produced by the Anastrepha Tra2 protein was, however, partial, indicating that the function of this protein in Drosophila was incomplete. There are two possible explanations for this. It might be due to an insufficient quantity of Anastrepha Tra2 protein being produced in the Drosophila transgenic flies; it was necessary to restrict the amount of Anastrepha Tra2 protein that was expressed since the production of any greater amount is lethal. Alternatively, the interaction between the endogenous DrosophilaTra protein and the transgenic Anastrepha Tra2 protein might be affected such that the DrosophilaTraAnastrephaTra2 complex is less efficient than the DrosophilaTra-Tra2 complex at inducing the female-specific splicing of the endogenous Drosophiladsxpre-mRNA, as it has been suggested for the interaction between the Anastrepha Tra and the Drosophila Tra2 proteins (Ruiz and Sánchez 2010). In this context, it is worth mentioning firstly the high degree of divergence
Fig. 2. Effect of the Aotra2 transgene on the splicing of Drosophila dsxpre-mRNA. (A) Molecular organisation of Drosophila dsx pre-mRNA showing the male and the female splicing pattern. Exons are represented in boxes and introns by dotted lines. The red bars in exon 4 represent the specific binding sequences for the Tra-Tra2 complex. (B) RT-PCR analyses of total RNA from D. melanogaster $X X$ pseudomales mutant for tra-2 and either not expressing $\left(25^{\circ} \mathrm{C}\right)$ or expressing (HS) the Aotra-2 transgene. $H S$ indicates heat shock treatment. TDAo\#2: XX pseudomale yw/w; tra-2B/Df(2R)trix, tra-2[-];TDAo\#2/hs-GAL4,w[+]; TDAo\#3: XX pseudomale yw/w; tra-2B/Df(2R)trix, tra-2[-]; TDAo\#3 / hsGAL4,w[+] (Crosses II and III in Materials and Methods). (C) RT-PCR analyses of total RNA from D. melanogaster XY males mutant for tra-2 (brothers of the XX pseudomales). As a control, the splicing of the primary transcript of gene rp49 that codes for the constitutive ribosomal protein 49 was monitored. 
between the Anastrepha and the Drosophila Tra2 proteins (Sarno et al., 2010); secondly, the incomplete feminisation of Drosophila $\mathrm{XX}$ pseudomales mutant for tra and expressing the Ceratitis Tra protein (Pane et al., 2005); and thirdly, the incomplete feminisation of Drosophila XX pseudomales mutant for tra-2 and expressing either the human (Dauwalder et al., 1996) or the Sciara (Martin et al., 2011) tra-2 ortholog, whereas Drosophila virilis tra-2 gene can fully replace the endogenous tra-2 function of Drosophila melanogaster for normal female sexual development (Chandler et al., 1997). Collectively, these results suggest that the interaction between the Tra and Tra2 proteins of different species might be impeded as a consequence of changes accumulated in these proteins after the drosophilids separated from the other dipteran phylogenetic lineages, thus suggesting that the Tra and Tra2 proteins co-evolved to exert their functions in sex determination.

\section{Materials and Methods}

\section{Flies and crosses}

Drosophila flies were cultured on standard food. For the description of the mutant alleles and GAL4 constructs see Lindsley and Zimm (1992) and FlyBase. The crosses to produce the flies were:

Cross I: Females ywTDAo\#1; tra-2B/CyO Cy; Tub-GAL80ts[w+]/MKRS,Sb and males $w / Y$; Df(2R)trix, tra-2[-]/CyO Cy; rn-GAL4[w+]/MKRS,Sb

Cross II: Females yw; tra-2B/CyO Cy; TDAo\#2[w+]/MKRS,Sb

and males $w / Y$; Df(2R)trix, tra-2[-]/CyOCy; hs-GAL4[ry+]/MKRS,Sb

Cross III: Females $y w$; tra-2B/CyO Cy; TDAo\#3[w+]/MKRS,Sb

and males $w / Y$; Df(2R)trix, tra-2[-]/CyO Cy; hs-GAL4[ry+]/MKRS, Sb

\section{Morphological analysis}

Flies used for the analysis of adult forelegs were kept in a mixture of ethanol:glycerol $(3: 1)$ for several days. They were then macerated in $10 \%$ $\mathrm{KOH}$ at $60^{\circ} \mathrm{C}$ for $15 \mathrm{~min}$, thoroughly washed with water and mounted in Faure's solution for inspection under a compound microscope.

\section{Production of the Aotra2 transgenic Drosophila melanogaster lines (TDAo)}

For the construction of the Aotra2 transgene, the tra-2 ORF of $A$. obliqua was amplified by RT-PCR. The PCR reaction was performed using primers PRtra2-1Ao (5'AGAGTTGGAATGAGTCCACG3') and PRtra2-2Ao (5'CATATTTTTAATAGCGCGTACG3'). The resulting amplicon was cloned in pGEMT-easy (Promega), following manufacturer's instructions. The inserted fragment was then cut with EcoRI and cloned in the EcoRI site of pUAST. The microinjections for generating the TDAo (UAS::Aotra-2cDNA) transgenic $D$. melanogaster lines were performed by Genetic Services (Sudbury, MA, USA). To ascertain that each transgenic line was carrying the correct transgene, PCR on genomic DNA with primers PRtra2-1Ao and PRtra2-2Ao was used to amplify the whole transgene and the amplicons were cloned in pGEMT-easy (Promega), following manufacturer's instructions and sequenced. Three transgenic lines were produced: TDAo\#1 (with the Aotra-2 transgene inserted in the X chromosome), and TDAo\#2 and TDAo\#3 with the Aotra-2 transgene inserted in the third chromosome. TDAo stands for $p U A S::$ Aotra-2 cDNA.

\section{Analysis of the splicing of dsx pre-mRNA in transgenic Drosophila Melanogaster lines}

The effect of AnastrephaTra2 protein on the splicing control of Drosophila $d s x$ pre-mRNA was studied in transgenic Drosophila XX flies mutant for tra-2 and expressing the Anastrepha Tra2 protein. The inducible hs-GAL4 driver was used to express the Aotra2 transgene. XX pseudomales of genotype $y w / w$; tra-2B/Df(2R)trix,tra2-; TDAo\#2/hs-GAL4 and of genotype $y w / w$; tra-2B/Df(2R)trix, tra2-; TDAo\#2/hs-GAL4, and their respective $\mathrm{XY}$ brothers of genotype $y w / Y$; tra-2B/Df(2R)trix,tra2-; TDAo\#2/hs-GAL4 and of genotype $y w / Y$; tra-2B/Df(2R)trix,tra2-; TDAo\#2/hs-GAL4 were produced at $25^{\circ} \mathrm{C}$ (see Crosses II and III above). After the hatching of the adults, the $\mathrm{XX}$ pseudomales and their $\mathrm{XY}$ brothers were divided into two populations; one was maintained at $25^{\circ} \mathrm{C}$ (control flies) and one subjected to heat-shock pulses (experimental flies) to induce the expression of the Aotra2 transgene. Two 3 hours heat shock pulses $\left(37^{\circ} \mathrm{C}\right)$ per day for two consecutive days with recovery at $25^{\circ} \mathrm{C}$ between pulses were given to the experimental flies. The effect of transgenic AoTra2 protein on Drosophila dsx pre-mRNA splicing was analysed by RT-PCR on total RNA from adults, which was prepared using the Ultraspec-II RNA isolation kit (Biotecx) following the manufacturer's instructions. Reverse transcription reactions were performed with an oligo-dT. Two percent of the synthesised cDNA was amplified by PCR, and one percent of this PCR was used for a second round of amplification, in a total volume of $50 \mu \mathrm{l}$. The primers used were: dsx1 (5'CATCGGGAACATCGGTGATC3') and dsxF1 (5'AACGGCTGTCACACATCGAC3').

The PCR conditions were $95^{\circ} \mathrm{C}, 5$ minutes, followed by 45 cycles of $95^{\circ} \mathrm{C}$ for 10 seconds, $60^{\circ} \mathrm{C}$ for 30 seconds, and $72^{\circ} \mathrm{C}$ for 20 seconds each cycle, plus an extension step at $72^{\circ} \mathrm{C}$ for 7 minutes. In all cases, PCR reactions with RNAsamples were performed to guarantee there was no contamination with genomic DNA (negative controls of PCR reactions). The amplicons were cloned in pGEMT-easy (Promega), following manufacturer's instructions.

\section{DNA sequencing}

It was used an automated 377 DNA sequencer (Applied Biosystems) and the primers forward M13 (-20) and reverse M13 reverse (-24).

\section{Acknowledgments}

We thank B. Oliver, I. Guerrero, M. Calleja, F. Díaz-Benjumea, E. Sánchez-Herrero and S. Campuzano for providing Drosophila stocks. We are grateful to F. Díaz-Benjumea for bringing our attention to the GAL-80 driver. This work was financed by grant BFU2008-00474 awarded to $L$. Sánchez by the D.G.I.C.Y.T.

\section{References}

BELL, L. R., HORABIN, J. I., SCHEDL, P. and CLINE, T.W. (1991). Positive autoregulation of Sex-lethal by alternative splicing maintains the female determined state in Drosophila. Cell 65: 229-239.

BELOTE. J. M., MCKEOWN, M., BOGGS, R. T., OHKAWA, R. and SOSNOWSKI B. A. (1989). The molecular genetics of transformer, a genetic switch-controlling sexual differentiation in Drosophila. Devel. Genet. 10: 143-154.

BOGGS, R. T., GREGOR, P., IDRISS, S., BELOTE, J. M. and MCKEOWN, M. (1987) Regulation of sexual differentiation in Drosophila Melanogaster via alternative splicing of RNA from the transformer gene. Cell 50: 739-747.

BURTIS, K.C. and BAKER, B.S. (1989). Drosophila doublesex gene controls somatic sexual differentiation by producing alternatively spliced mRNAs encoding related sex-specific polypeptides. Cell 56: 997-1010.

BURGHARDT, G., HEDIGER, M., SIEGENTHALER, C., MOSER, M., DÜBENDORFER, A and BOPP, D. (2005) The transformer-2 gene in Musca domestica is required for selecting and maintaining the female pathway of development. Dev Genes Evol 215:165-176.

CHANDLER, D., MCGUFFIN, M., PISKUR, J., YAO, J., BAKER, B. and MATTOX, W. (1997) Evolutionary conservation of regulatory strategies for the sex determination factor transformer-2. Mol Cell Biol 17: 2908-2919.

CONCHA, C. and SCOTT, M. J. (2009) Sexual development in Lucilia cuprina (Diptera, Calliphoridae) is controlled by the transformergene. Genetics 182: 785-789.

DAUWALDER, B., AMAYA-MANZANARES, F. AND MATTOX, W. (1996) A human homologue of the Drosophila sex determination factor transformer-2 has conserved splicing regulatory functions. Proc. Natl. Acad. Sci. USA 93: 9004-9009.

GEMPE, T and BEYE, M. (2010). Function and evolution of sex determination mechanisms, genes and pathways in insects. BioEssays 33: 52-60.

HEDIGER, M., HENGGELER, C., MEIER, N., PÉREZ, R., SACCONE, G. and BOPP, D. (2010) Molecular characterization of the key switch $F$ provides a basis for understanding the rapid divergence of the sex-determining pathway in the 
housefly. Genetics 184: 155-170.

HEDLEY, M. and MANIATIS, T. (1991) Sex-specific splicing and polyadenylation of $d s x$ pre-mRNA requires a sequence that binds specifically to tra-2 protein in vitro. Cell 65: 579-586.

HOSHIJIMA, K., INOUE, K., HIGUCHI, I., SAKAMOTO, H. and SHIMURA, Y. (1991). Control of doublesex alternative splicing by transformer and transformer-2 in Drosophila. Science 252: 833-836.

LAGOS, D., RUIZ, M. F., SÁNCHEZ, L. and KOMITOPOULOU, K. (2005) Isolation and characterization of the Bactrocera oleaegenes orthologous to the sex determining Sex-lethal and doublesex genes of Drosophila Melanogaster. Gene 384:111-121.

LAGOS, D., KOUKIDOU, M., SAVAKIS, C. and KOMITOPOULOU, K. (2007). The transformer gene in Bactrocera oleae: the genetic switch that determines its sex fate. Insect Mol. Biol. 16: 221-230.

LINDSLEY, D. L. and ZIMM, G.(1992) The genome of Drosophila Melanogaster. San Diego, California. Academic Press.

MARTÍN, I., RUIZ, M. F. AND SÁNCHEZ, L. (2011) The gene transformer-2 of Sciara (Diptera, Nematocera) and its effect on Drosophila sexual development. BMC Dev. Biol. 11: 19.

MCGUIRE, S. E., LE, P. H., OSBORN, A. J., MATSUMOTO, K. and DAVIS, R. L. (2003). Spatiotemporal rescue of memory dysfunction in Drosophila. Science 302: 1765-1768.

PANE, A., SALVEMINI, M., BOVI, P. D., POLITO, C. and SACCONE, G. (2002). The transformer gene in Ceratitis capitata provides a genetic basis for selecting and remembering the sexual fate. Development 129: 3715-3725.

PANE, A., DE SIMONE, A., SACCONE, G and POLITO, C. (2005). Evolutionary conservation of Ceratitis capitata transformer gene function. Genetics 171: 615-624.

QI, J., SU, S., MCGUFFIN, M. E. and MATTOX, W. (2006). Concentration dependent selection of targets by an SR splicing regulator results in tissue-specific RNA processing. Nucl. Acids Res. 34: 6256-6263.

RUIZ, M. F., MILANO, A., SALVEMINI, M., EIRÍN-LÓPEZ, J.M., PERONDINI, A. L.
P., SELIVON, D., POLITO, C., SACCONE, G. and SÁNCHEZ, L. (2007). The gene transformer of Anastrepha fruit flies (Diptera, Tephritidae) and its evolution in insects. PLoS ONE 2: e1239.

RUIZ, M. F. and SÁNCHEZ, L. (2010). Effect of the gene transformer of Anastrepha on the somatic sexual development of Drosophila. Int. J. Dev. Biol. 54: 627-633.

RYNER, L. C. and BAKER, B. S. (1991). Regulation of doublesex pre-mRNA processing occurs by 3'-splice site activation. Genes Dev. 5: 2071-2085.

SACCONE, G., PELUSO, I., ARTIACO, D., GIORDANO, E., BOPP, D. and POLITO, C. (1998). The Ceratitis capitata homologue of the Drosophila sex-determining gene Sex-lethal is structurally conserved, but not sex-specifically regulated. Development 125: 1495-1500.

SALVEMINI, M., ROBERTSON, M., ARONSON, B., ATKINSON, P., POLITO, C and SACCONE, G. (2009). Ceratitis capitata transformer-2 gene is required to establish and maintain the autoregulation of Cctra, the master gene for female sex determination. Int. J. Dev. Biol. 53: 109-120.

SÁNCHEZ, L., GORFINKIEL, N. and GUERRERO, I. (2005). Sex determination and the development of the genital disc. In Comprehensive Molecular Insect Science, Volume 1, pp. 1-38. Edited by Gilbert LI, latrou K, Gill SS. Oxford, Elsevier Pergamon.

SÁNCHEZ, L. (2008). Sex-determining mechanisms in insects. Int. J. Dev. Biol. 52 1059-1075.

SARNO, F., RUIZ, M. F., EIRÍN-LÓPEZ, J. M., PERONDINI, A. L. P., SELIVON, D. and SÁNCHEZ, L. (2010). The gene transformer-2 of Anastrepha fruit flies (Diptera, Tephritidae) and its evolution in insects. BMC Evol Biol 10: 140.

ST. PIERRE, S. E., GALINDO, M. I., COUSO, J. P. and THOR, S. (2002). Control of Drosophila imaginal disc development by rotund and roughened eye, differentially expressed transcripts of the same encoding functionally distinct zinc finger proteins. Development 129: 1273-1281.

VERHULST, E. C., VAN DE ZANDE, L. and BEUKEBOOM, L. W. (2010) Insect sex determination: it all evolves around transformer. Curr. Opin. Gene. Devel. 20: 1-8. 


\section{Further Related Reading, published previously in the Int. J. Dev. Biol.}

Effect of the gene transformer of Anastrepha on the somatic sexual development of Drosophila

Ruiz MF and Sánchez L.

Int. J. Dev. Biol. (2010) 54: 627-633

Genetic evidence against a role for W-linked histidine triad nucleotide binding protein (HINTW) in avian sex determination Craig A. Smith, Kelly N. Roeszler and Andrew H. Sinclair.

Int. J. Dev. Biol. (2009) 53: 59-67

Ceratitis capitata transformer-2 gene is required to establish and maintain the autoregulation of Cctra, the master gene for female sex determination

Salvemini M, Robertson M, Aronson B, Atkinson P, Polito LC and Saccone G.

Int. J. Dev. Biol. (2009) 53: 109-120

Cloning and functional characterization of the intersex homologous gene in the pest lepidopteron Maruca vitrata Cavaliere D, di Cara F, Polito LC, and digilio FA.

Int. J. Dev. Biol. (2009) 53: 1057-1062

Sex-determining mechanisms in insects

Sánchez L.

Int. J. Dev. Biol. (2008) 52: 1059-1075

Masculinization of XX Drosophila transgenic flies expressing the Ceratitis capitata DoublsexM isoform

Saccone G, Salvemini M, Pane A and Polito C.

Int J Dev Biol (2008) 52: 1043-1050

Musca domestica, a window on the evolution of sex-determining mechanisms in insects. Dübendorfer A, Hediger M, Burghardt G and Bopp D.

Int. J. Dev. Biol. (2002) 46: 75-79

In vitro binding and expression studies demonstrate a role for the mouse Sry Q-rich domain in sex determination

$\mathrm{J}$ Zhang J, Coward, Xian M and Lau YF.

Int. J. Dev. Biol. (1999) 43: 219-227

Characterization of ovine SRY transcript and developmental expression of genes involved in sexual differentiation

Payen E, Pailhoux E, Abou Merhi R, Gianquinto L, Kirszenbaum M, Locatelli A and Cotinot C. Int. J. Dev. Biol. (1996) 40: 567-575

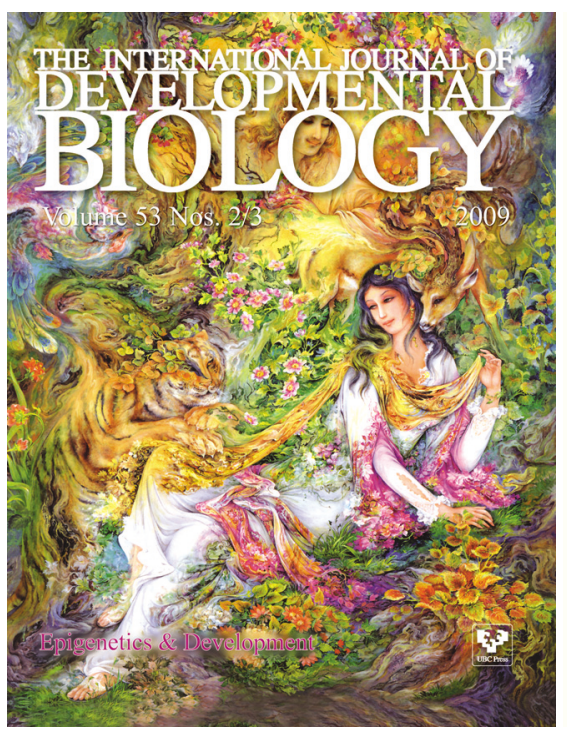

ISI Impact Factor $(\mathbf{2 0 1 0})=2.86$
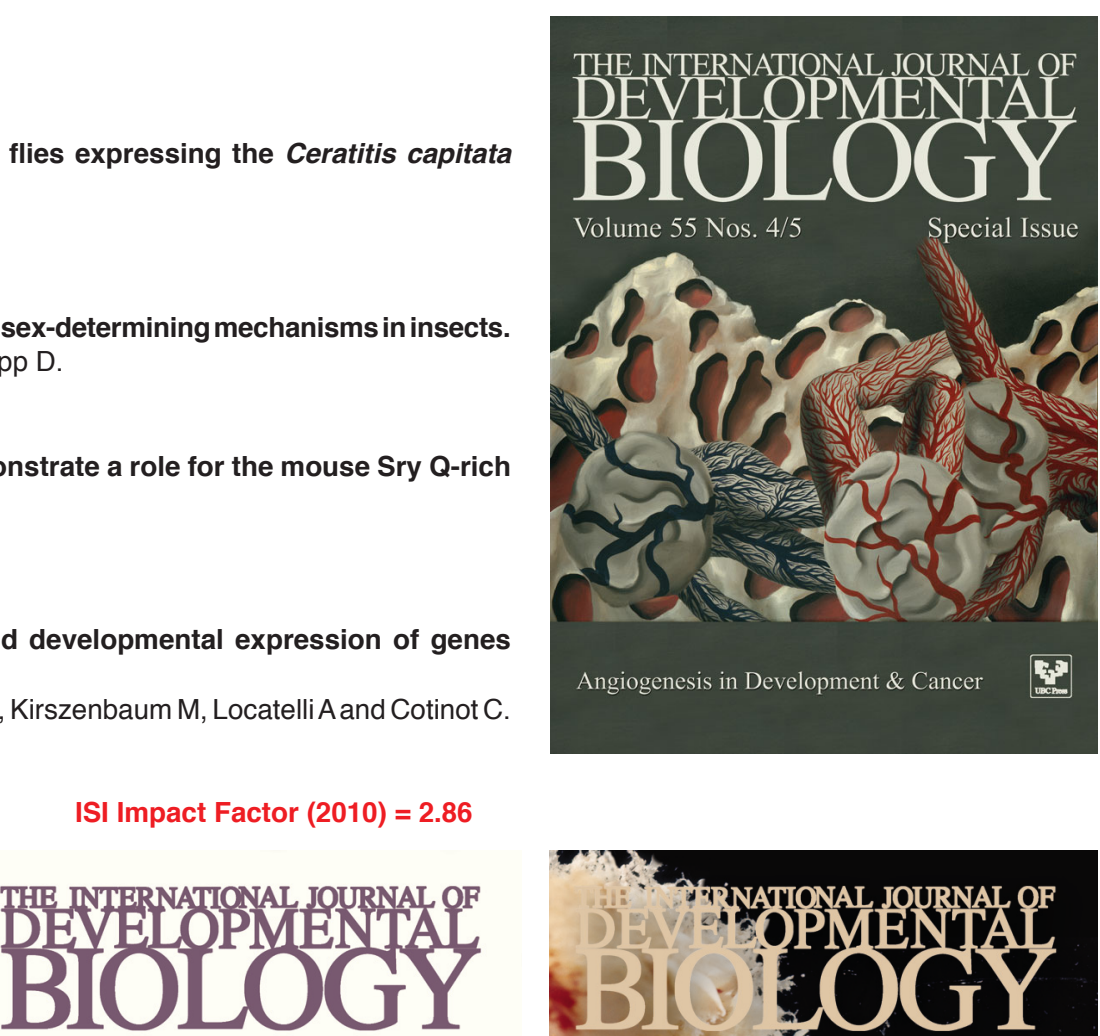

Volume 54 Nos. 6/7

Special Issue
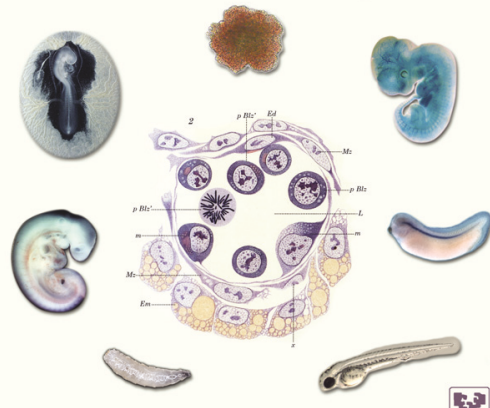

Developmental Hematopoiesis
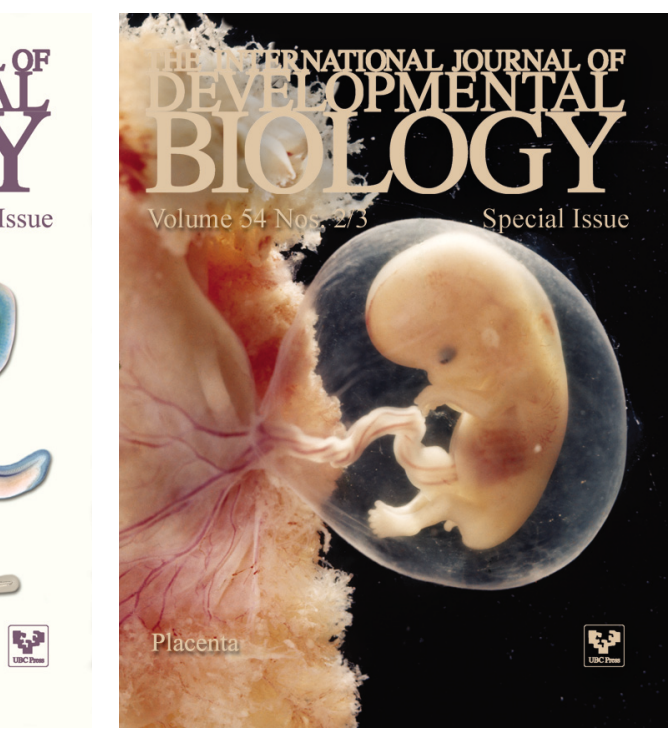